\title{
Differing effects of systemically administered rapamycin on consolidation and reconsolidation of context vs. cued fear memories
}

\author{
Ebony M. Glover, ${ }^{1}$ Kerry J. Ressler, and Michael Davis \\ Department of Psychiatry and Behavioral Sciences, Center for Behavioral Neuroscience, Emory University, Atlanta, Georgia 30302, \\ USA; Yerkes National Primate Research Center, Emory University, Atlanta, Georgia 30329, USA
}

\begin{abstract}
Rapamycin, an inhibitor of the mammalian target of rapamycin (mTOR) kinase, has attracted interest as a possible prophylactic for post-traumatic stress disorder (PTSD)-associated fear memories. We report here that although rapamycin ( $40 \mathrm{mg} / \mathrm{kg}$, i.p.) disrupted the consolidation and reconsolidation of fear-potentiated startle paradigm to a shock-paired context, it did not disrupt startle increases to a 4-sec odor cue, even though post-training increases in amygdala mTOR activity were prevented by rapamycin (also $40 \mathrm{mg} / \mathrm{kg}$, i.p.). Thus, while rapamycin may prove useful in retarding the development of some PTSD-associated memories, its relative ineffectiveness against cued fear memories may limit its clinical usefulness.
\end{abstract}

Disrupting post-trauma fear memories may be a useful approach for mitigating post-traumatic stress disorder (PTSD). Preclinical and clinical evidence shows that various manipulations, when administered shortly after learning (c.f., McGaugh 2000) or retrieval (e.g., Misanin et al. 1968; Nader et al. 2000) can induce retrograde amnesia, thus providing a possible approach to PTSD treatment.

The mammalian target of rapamycin (mTOR) kinase modulates phosphorylation of the $70-\mathrm{kDa}$ ribosomal S6 kinase (p70s6K), which regulates protein translation (Raught et al. 2001). mTOR signaling has been implicated in synaptic plasticity (Casadio et al. 1999; Tang et al. 2002; Cammalleri et al. 2003) and learning and memory (Tischmeyer et al. 2003; Parsons et al. 2006; Bekinschtein et al. 2007; Blundell et al. 2008) and, indeed, rapamycin, an inhibitor of mTOR, disrupts the consolidation and reconsolidation of tone-shock as well as context-shock fear memories (assessed with freezing) when administered directly into the amygdala of rats (Parsons et al. 2006). Importantly, rapamycin has also been shown to disrupt the consolidation and reconsolidation of context-shock memories (also assessed with freezing) when given systemically (Blundell et al. 2008). That systemic rapamycin administration disrupts fear memories in rodents makes it a promising tool in the pharmacotherapeautic treatment of PTSD. Furthermore, rapamycin is FDA-approved for use in humans, and is already widely prescribed for various conditions (Plas and Thomas 2009).

To evaluate the generality of systemic rapamycin effects on fear memory consolidation and reconsolidation, and hence the potential therapeutic efficacy of this drug, we examined the effects of post-training and post-recall systemic rapamycin injections on single-trial odor-shock and context-shock fear memories in rats. To assess Pavlovian fear memory of both, we used a fearpotentiated startle (FPS) protocol in which rats show an increased noise-elicited startle response in the presence of an odor conditioned stimulus (CS) that had been previously paired with a footshock unconditioned stimulus (US) (Paschall and Davis 2002), and to the context where odor-shock conditioning occurred (McNish et al. 1997; McNish et al. 2000).

'Corresponding author.

E-mail eglover@emory.edu; fax (404) 727-8070.

Article is online at http://www.learnmem.org/cgi/doi/10.1101//m.1908310.
Elevated phosphorylated p70s6K has been observed in the amygdala after tone-shock and context-shock conditioning, and these changes are prevented by intra-amygdala rapamycin infusions (Parsons et al. 2006). To determine if systemic rapamycin has similar effects, we also assessed the effect of single-trial olfactory fear conditioning, with and without immediate post-training systemic rapamycin injections, on amygdala levels of phosphorylated p70s6K.

Male Sprague-Dawley rats $(N=126)$ (Charles River, NC), weighing 350-400 g and group housed four to a cage, were used for these experiments. Rats were trained and tested in two identical cages as previously described (Cassella and Davis 1986; Paschall and Davis 2002). The CS was a discrete 4 -sec odor (5\% amyl acetate), and the US was a 0.5 -sec, $0.4-\mathrm{mA}$ footshock. On two consecutive days, rats received 30 presentations of startleeliciting 95-dB noise bursts (30-sec interstimulus interval [ISI]). Mean startle amplitudes were calculated and used as the pretraining startle baseline. The next day, rats received a single odorshock pairing. Immediately thereafter, rats in the Consolidation group were given a systemic injection (i.p.) of either rapamycin $(40 \mathrm{mg} / \mathrm{kg})$ or vehicle, whereas rats in the Reconsolidation group were returned to their home cage and then, $24 \mathrm{~h}$ later, presented again with a single 4-sec odor CS (but without shock) followed by either rapamycin $(40 \mathrm{mg} / \mathrm{kg})$ or vehicle. This dose was chosen based on a previous dose-response study by Blundell et al. (2008), which demonstrated that $40 \mathrm{mg} / \mathrm{kg}$ of rapamycin was most effective at disrupting context-shock fear memories, while having no effects on locomotor activity or pain sensitivity. For both groups, rapamycin (LC Laboratories), was dissolved in a vehicle made of 5\% ethanol, 4\% PEG400, 4\% Tween 80, and sterile water. Both drug and vehicle were delivered in a volume of $0.8 \mathrm{~mL} / 100 \mathrm{~g}$ body weight.

Seven days after training or reactivation, rats were tested for FPS. After 5 min of habituation to the chamber, rats were presented with 30 startle-eliciting noise bursts. Thirty seconds after the final startle stimulus, rats received 30 startle stimuli presented alone (noise-alone trial) and 10 noise bursts presented $3.2 \mathrm{sec}$ after onset of the 4-sec odor (odor-noise trials). The two trial types were presented in a manner such that after each odor-noise trial, three noise-alone trials were presented at a 30 -sec ITI, so that noise-alone trials occurred 30, 60, and $90 \mathrm{sec}$ after each odor. For some rats, 
training and test contexts were identical. For others, the test context was altered (i.e., sandpaper inserts over the shock bars, Velcro on the sides, and two metal-link chains suspended from the top). These alterations have been previously shown to reliably produce discriminable context conditioning (McNish et al. 1997).

For Western blot analyses, a separate group of rats was presented with a single odor presentation that was either Paired (odor co-terminated with footshock) or Unpaired (odor and footshock separated by a 2-min interval) with shock and then euthanized via decapitation either 30 or 60 min later. Another cohort was trained with a single odor-shock pairing, and immediately thereafter given i.p. injections of either rapamycin $(40 \mathrm{mg} / \mathrm{kg})$ or vehicle, then sacrificed $30 \mathrm{~min}$ later. Control groups were placed in the conditioning chamber for $7 \mathrm{~min}$, without odor or shock exposure and sacrificed $1 \mathrm{~h}$ later, or were never exposed to the conditioning chamber.

Brains were removed rapidly and the basolateral nucleus of the amygdala (BLA) excised bilaterally using a brain punch, homogenized in buffer ( $5 \mathrm{mM}$ HEPES, $0.32 \mathrm{M}$ sucrose, and Complete Mini EDTA-free protease inhibitor, Roche Diagnostics), and stored at $-80^{\circ} \mathrm{C}$. Protein samples were quantified using a standard BCA assay (Pierce). Equal quantities $(5-10 \mu \mathrm{g})$ of protein per animal were loaded onto polyacrilamide-SDS mini-gels, separated electrophoretically, blotted onto nitrocellulose membranes (BioRad), blocked, and incubated overnight at $4{ }^{\circ} \mathrm{C}$ in primary for phosphop70s6K (Thr 412) (1:1000; Upstate Biotechnology). After primary antibody exposure, membranes were incubated in a secondary antibody (1:5000; Upstate Biotechnology) for $90 \mathrm{~min}$. Bound antibody was detected by SuperSignal West Chemiluminescence (Pierce) using a fluorchem imaging system (Alpha Innotech). Antibody detection of glyceraldehyde-3-phosphate dehydrogenase (GAPDH: mouse monoclonal, 1:10,000, Research Diagnostics) was used to control for variations in protein loading.

For statistical analyses of FPS to the odor cue, the mean startle amplitude on noise-alone and odor-noise test trials was determined for each rat and entered into an ANOVA with Trial Type as a repeated measure and Treatment (rapamycin or vehicle) as a between-subjects measure. For context fear the mean startle amplitude of the 30 noise-alone trials of the final pre-conditioning acclimation session and the mean startle amplitude of the
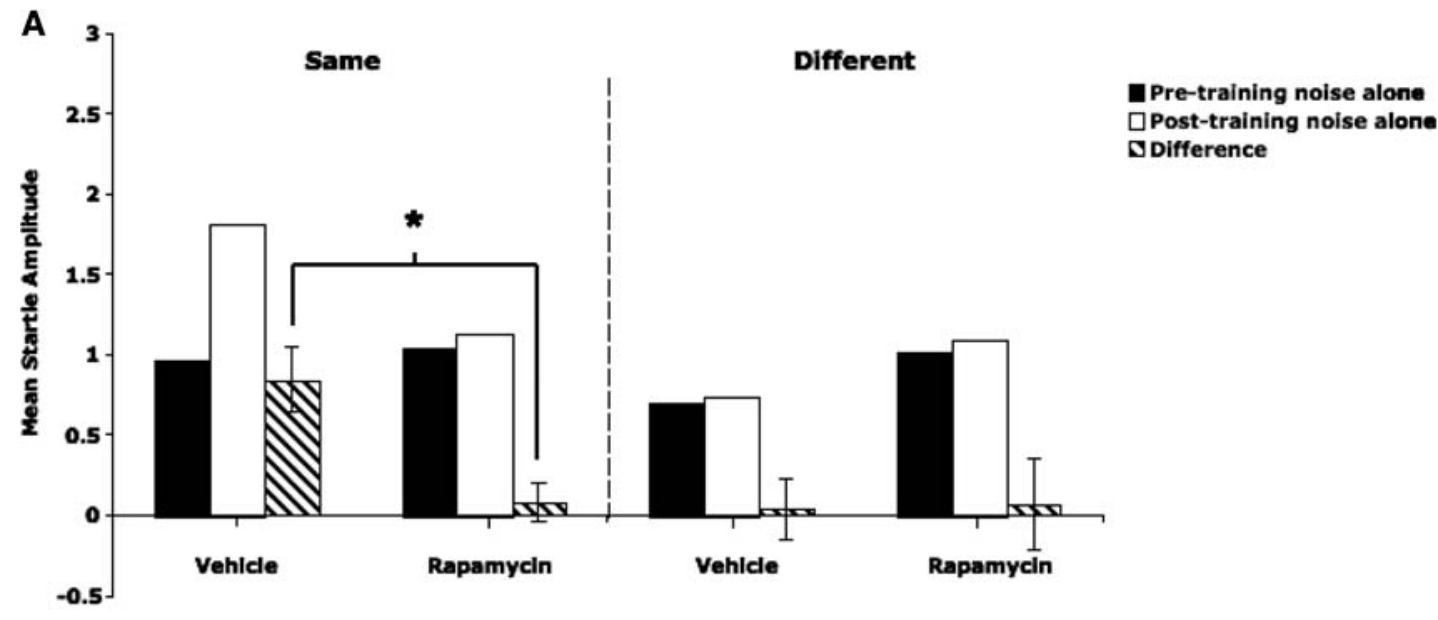

DDifference

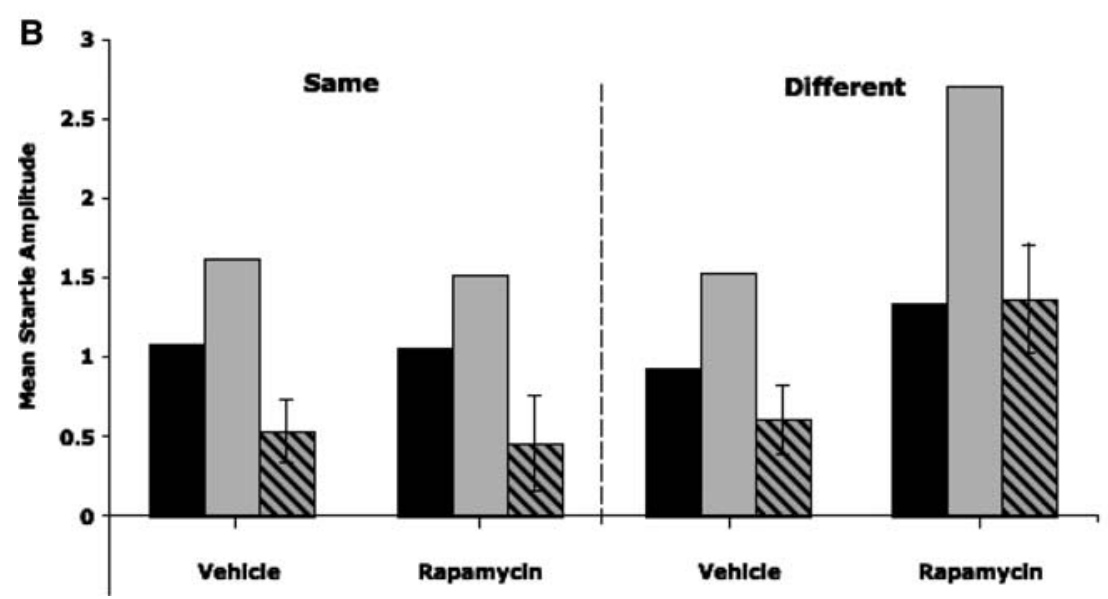

Figure 1. Systemic rapamycin disrupts the consolidation of context but not cued fear memory. Mean startle amplitudes of rats given an i.p. injection of either rapamycin $(40 \mathrm{mg} / \mathrm{kg})$ or vehicle immediately after training, and tested in the same context in which they were trained or tested in a different context. (A) Context Fear, mean startle amplitude for pre-training noise-alone (black), and post-training noise-alone (white) trials, and the difference ( \pm SEM) between the two (black and white stripes). Rapamycin-treated rats showed significantly less FPS to the conditioning context than vehicle-treated rats $\left({ }^{*} P<0.05\right)$. None of the rats that were tested in a different context expressed context fear memory. (B) Cued Fear, mean startle amplitude for posttraining noise-alone (dark gray), post-training odor-noise (light gray), and the difference ( \pm SEM) between the two (gray and black stripes). There was no significant difference between rapamycin and vehicle-treated rats in their expression of cued fear memory whether tested in the same or a different context from training. 
30 noise-alone trials that occurred at the beginning of the postconditioning test session were determined and also entered into an ANOVA with Session as a repeated measure and Treatment (rapamycin or vehicle) as a between-subjects measure. For Western blotting analyses, a percentage of control score was derived for each rat by dividing each animal's relative optical density score by the home cage control group mean. Data were analyzed by analysis of variance (ANOVA) or repeated measures followed by individual mean comparison using Tukey's post-hoc tests. A significance level of $P<0.05$ was taken for all results.

We first examined the effects of systemically administered rapamycin on the consolidation of Pavlovian fear conditioning to the odor and context cues. As shown in Figure 1, systemic rapamycin significantly disrupted the long-term memory of context fear, but not cued fear. For animals trained and tested in the same context, rapamycin prevented the increase in startle amplitude that occurred from the pre- to post-conditioning test session noise-alone trials (i.e., context fear). This was confirmed by a significant Session $\times$ Treatment interaction, $F_{(1,16)}=4.57, P<0.05$.
Animals that were tested in a different context did not express context fear memory, as indicated by the lack of a significant main effect of Session, $F_{(1,31)}=0.004, P>0.05$, or Session $\times$ Treatment interaction, $F_{(1,31)}=0.06, P>0.05$.

As shown in Figure $1 \mathrm{~B}$, rapamycin did not prevent the increase in startle that occurred on odor-noise trials. For animals trained and tested in the same context, this was indicated by a significant main effect of Trial Type (noise-alone vs. odor-noise trials), $F_{(1,16)}=11.89, P<0.05$, without a significant Trial Type $\times$ Treatment interaction, $F_{(1,16)}=0.29, P>0.05$. For animals trained and tested in a different context, the results were similar with a significant main effect of Trial Type, $F_{(1,31)}=31.91, P<$ 0.05 , without a significant Trial Type $\times$ Treatment interaction $F_{(1,31)}=4.73, P>0.05$. Thus, rapamycin blocked the consolidation of context but not cued FPS.

We next tested the effects of systemic rapamycin on the reconsolidation of context and cued fear memory. For animals trained and tested in the same context (Fig. 2A), rapamycin prevented the increase in startle amplitude that occurred from the
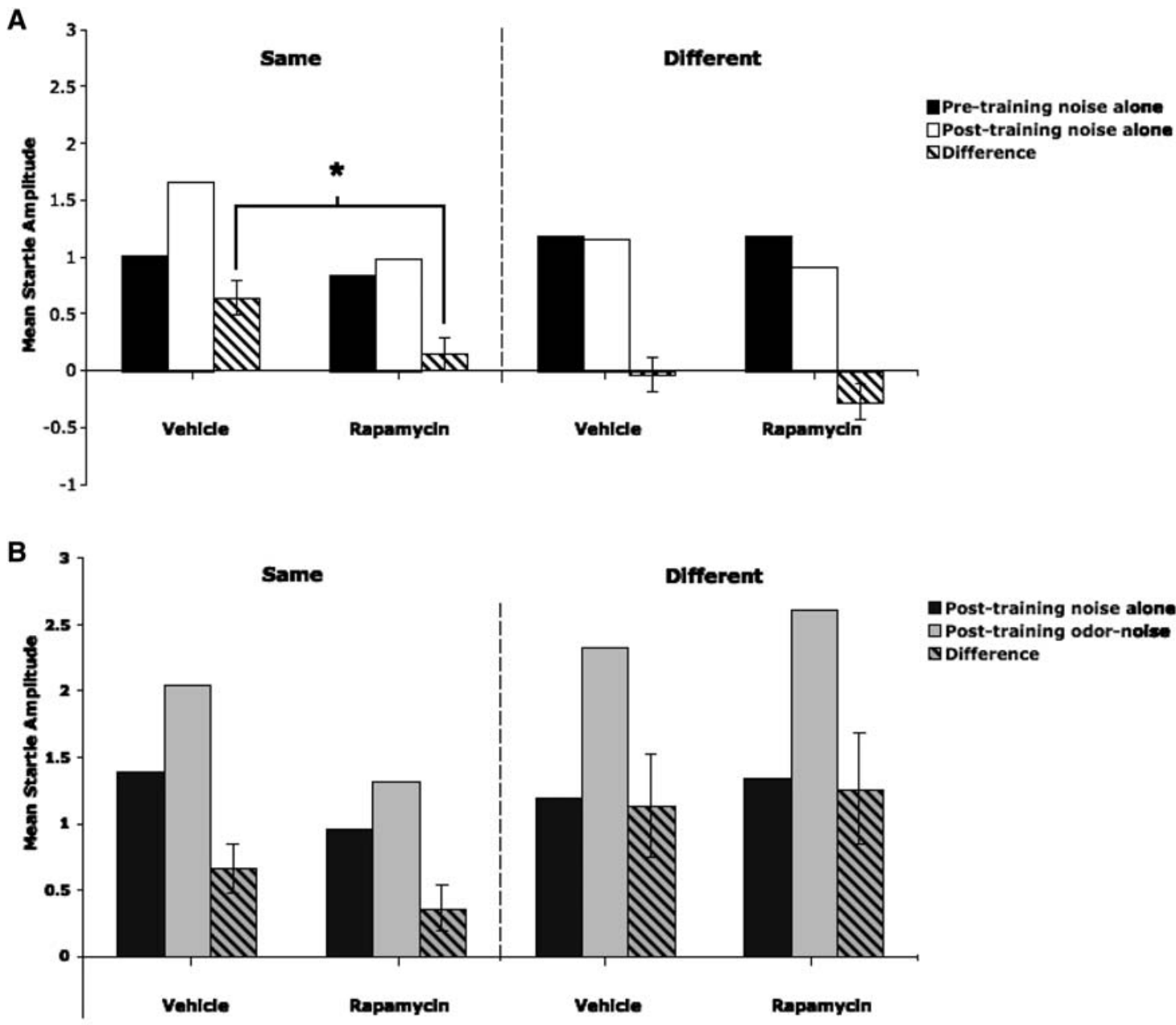

$\square$ Post-training noise alone $\square$ Post-training odor-nolse Difference

Figure 2. Systemic rapamycin disrupts the reconsolidation of context but not cued fear memory. Mean startle amplitudes of rats given an i.p. injection of either rapamycin $(40 \mathrm{mg} / \mathrm{kg})$ or vehicle immediately after memory reactivation, and tested in the same context in which they were trained or tested in a different context. $(A)$ Context Fear, mean startle amplitude for pre-training noise-alone (black), and post-training noise-alone (white) trials $6 \mathrm{~d}$ after memory reactivation and the difference ( \pm SEM) between the two (black and white stripes). Rapamycin-treated rats showed significantly less FPS to the conditioning context than vehicle-treated rats $\left({ }^{*} P<0.05\right)$. None of the rats that were tested in a different context expressed context fear memory. $(B)$ Cued Fear, mean startle amplitude for post-training noise-alone (dark gray), post-training odor-noise (light gray), and the difference ( \pm SEM) between the two (gray and black stripes) $6 \mathrm{~d}$ after memory reactivation. There was no significant difference between rapamycin and vehicle treated rats in their expression of cued fear memory whether tested in the same or a different context from training. 
pre- to post-conditioning test session (i.e., context fear), as indicated by a significant Session $\times$ Treatment interaction $F_{(1,9)}=$ 6.27, $P<0.05$. Animals that were trained and tested in different contexts did not show context-potentiated startle, as indicated by the lack of a significant effect of Session, $F_{(1,8)}=2.00, P>$ 0.05 , or a significant Session $\times$ Treatment interaction, $F_{(1,8)}=$ $1.15, P>0.05$

The effects on olfactory FPS are shown in Figure 2B. For animals trained and tested in the same context (left panel) there was a significant main effect of Trial Type, $F_{(1,9)}=6.98, P<0.05$. It appears from this graph that rapamycin may have reduced startle in both the noise-alone and odor-noise trials. However, there was not a significant Trial Type $\times$ Treatment interaction, $F_{(1,9)}=0.08$, $P>0.05$, nor a significant main effect of Treatment, $F_{(1,9)}=0.005$, $P>0.05$. For animals trained and tested in a different context (right panel), there was a significant main effect of Trial Type, $F_{(1,8)}=17.53, \quad P<0.05$, but not a significant Trial Type $\times$ Treatment interaction, $F_{(1,8)}=0.05, P>0.05$. Similar to rapamycin effects on initial consolidation then, rapamycin blocked the reconsolidation of context but not cued FPS. Figure $3 \mathrm{~A}$ shows quantitative levels of phosphorylated p70s6K protein expressed relative to home cage and GAPDH loading control. The results show a clear increase in phosphorylated p70s6K relative to home cage controls in the paired group at the 30-min posttraining time point, and a moderate enhancement at the 1-h time point. Similarly, a modest increase was observed in the unpaired groups at both time points, probably as a result of context conditioning which often occurred with unpaired CS-US training. Nonexposed and home cage controls showed a very similar expression profile. An overall ANOVA comparing all groups approached but did not reach statistical significance, $F_{(5,29)}=$ $2.08, P<0.09$. A Tukey's post-hoc analysis revealed that the difference between home cage and paired 30 groups approached but did not reach significance, $P<0.08$. Although there clearly was a trend for increased phosphorylated p70s6K after one-trial conditioning, there was considerable variability in the data. In the next experiment, in which we utilized fresher antibody and loading gels, this variance was greatly reduced.

The effects of immediate post-training rapamycin $(40 \mathrm{mg} /$ $\mathrm{kg}$ ) on the relative increase in phosphosphorylated p70s6K seen 30 min after training are shown in Figure 3B. There was a significant increase in phosphorylated p70s6 K relative to home cage controls in the vehicle group, and diminished levels in the rapamycin group. An overall ANOVA comparing home cage, vehicle, and rapamycin groups revealed a significant difference among groups, $F_{(3,22)}=5.04, P<0.05$. A Tukey's post-hoc test revealed a significant difference between home cage and vehicle, $P<$ 0.05 , and vehicle and rapamycin, $P<0.05$, groups. These results support our previous finding that single-trial olfactory fear conditioning produces enhanced expression of phospho-p70s6 $\mathrm{K}$ in the amygdala $30 \mathrm{~min}$ after training and show further that systemic rapamycin prevents this increase.

Consistent with the findings of Blundell et al. (2008), who used freezing as a measure of fear, the current results show that systemic rapamycin disrupts both the consolidation and reconsolidation of context fear memory as measured by FPS. However, systemic rapamycin had no effect on the consolidation or the reconsolidation of cued fear memory. These findings are inconsistent with those of Parsons et al. (2006) who demonstrated that rapamycin disrupted both the consolidation and reconsolidation of cued (tone-shock) as well as context fear memories (assessed with freezing) in rats when administered locally into the amygdala (Parsons et al. 2006). This discrepancy may reflect the different modalities used in these two studies (i.e., tone vs. odor), different measures of fear (freezing vs. FPS), or a greater sensitivity
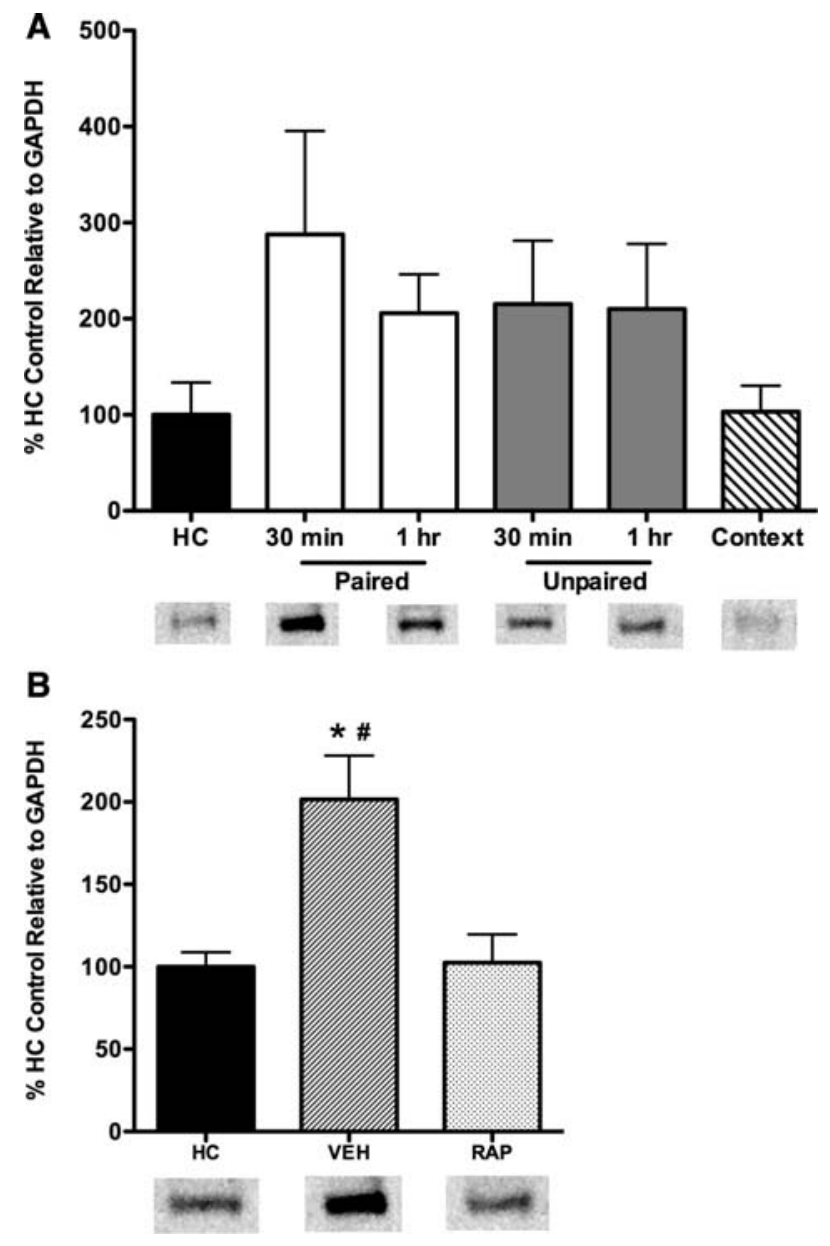

Figure 3. Single trial olfactory fear conditioning activates phosphorylation of p70s6K in the amygdala, which was prevented by post-training systemic rapamycin administration. Bars represent mean optical density values ( \pm SEM) expressed as a percentage of home cage $(H C)$, control (black), and GAPDH protein loading control. Representative blots of phosphorylated p70s6K are shown below their corresponding group. (A) Rats were presented with an odor cue and footshock that were either Paired (white) or Unpaired (gray), and were sacrificed $30 \mathrm{~min}$ or $60 \mathrm{~min}$ later. Another group, Context, was placed in the conditioning chamber for $7 \mathrm{~min}$, without odor or shock exposure, and sacrificed $1 \mathrm{~h}$ later (striped). Olfactory fear conditioning is associated with an increase in phosphorylated p70s6K relative to $\mathrm{HC}$, in the paired group at the 30 min post-training time point, as well as moderate enhancement at the 1-h time point, and in the unpaired groups at both 30-min and 1-h time points. These observed changes approached but did not reach statistical significance, $P=0.08$. (B) Immediately after single-trial odor-shock training, rats were given i.p. rapamycin (RAP, striped) $(40 \mathrm{mg} / \mathrm{kg})$ or vehicle (VEH, dotted) and sacrificed $30 \mathrm{~min}$ later. There was significant increase in phosphorylated p70s6K relative to HC in the VEH group $\left({ }^{*} \mathrm{P}<0.05\right.$ compared to $\left.\mathrm{HC}\right)$, and significantly less expression levels in the RAP group ( $\# P<0.05$ compared to VEH and $H C$ ).

of discrete cue fear conditioning to intra-amygdala vs. systemic rapamycin administration.

Using Western blot analysis, single trial olfactory fear conditioning resulted in enhanced expression of phosphorylated p70s6K in amygdala neurons (Fig. 3A,B). Moreover, an amnesic rapamycin dose $(40 \mathrm{mg} / \mathrm{kg})$ reduced p70s6K signaling when measured 30 min after training (Fig. 3B) similar to that found after local infusion of rapamycin into the amygdala (Parsons et al. 
2006). Hence, a systemic dose of rapamycin (40 mg/kg)—which robustly disrupted context fear memory-significantly reduced amygdala mTOR signaling, despite having no effect on the behavioral expression of cued fear. Overall, our findings suggest for the first time that mTOR signaling in the amygdala can be activated after one-trial olfactory fear conditioning, which may be relevant for context fear. However, its role in cued fear remains unclear. Because our single-trial olfactory conditioning paradigm simultaneously produced cued fear and context fear, our interpretation of amygdala p70s6K expression after training, and rapamycin's effect on this expression, is limited by the inability to distinguish cued fear specific vs. context fear specific p70s6K expression. Nevertheless, a previous study has found comparable levels of amygdala p70s6K expression after tone-shock and context-shock conditioning (Parsons et al. 2006). However, there are no studies to date that explicitly show the effects of rapamycin on amygdala mTOR signaling after context-shock conditioning absent of a discrete cue. Further research is needed to address this issue.

Overall the present study reports differential vulnerabilities of cued and context fear memories to systemic rapamycin treatment. These findings are consistent with previous research, which altogether show that systemically administered agents disrupt the consolidation of hippocampal-dependent (i.e., context fear or inhibitory avoidance), but not hippocampal-independent (i.e., cued fear) forms of fear memories (Lee et al. 2001; Debiec and Ledoux 2004; Grillon et al. 2004; Thomson and Sutherland 2005). It is not clear why the consolidation and reconsolidation of cued fear is impervious to systemic rapamycin treatment. It is possible that certain forms of fear memories might have a higher threshold for disruption than other forms. Further research could address this issue by doing a dose-response measure of systemic rapamycin on cued fear memory consolidation. It is possible that a different dose of rapamycin might be more efficacious in disrupting cued fear memory. However, our finding that $40 \mathrm{mg} /$ $\mathrm{kg}$ of rapamycin prevented the increase in amygdala mTOR signaling seen after cued fear conditioning suggests that dosage, by itself, may not be the critical issue. There is also the possibility that timing may be a critical factor. Cued fear memory's sensitivity to systemic rapamycin might be restricted to a certain temporal window. We injected rapamycin within $30 \mathrm{sec}$ of the odor-shock presentation or reactivation trial, and while this regimen was sufficient to disrupt amygdala mTOR signaling as well as longterm memory of context fear, it is possible that the critical learning-related changes that support long-term cued fear memory had already occurred.

Clearly, more research is needed to address these issues. Based on our findings, we conclude that although systemically administered mTOR inhibitors may prove useful in retarding the development of some PTSD-associated memories, their possible selectivity for disrupting context vs. cued fear memories might limit their clinical effectiveness.

\section{Acknowledgments}

We thank Georgette Gafford for her generous help in conducting Western blot experiments. This research was supported in part by NIMH Grants MH 47840 (R7) to M.D., MH884697 (RC1) to K.J.R., and the NIMH MH 542000 Mental Health Dissertation Grant to Increase Diversity (R36) granted to E.M.G. Portions of this work were submitted by E.M.G. to the Department of Psychology, Emory University, in partial fulfilment of the requirements for the doctoral degree. Animals used in this study were cared for in accordance with guidelines of the Emory University Institutional Animal Care and Use Committee.

\section{References}

Bekinschtein P, Katche C, Slipczuk LN, Igaz LM, Cammarota M, Izquierdo I, Medina JH. 2007. mTOR signaling in the hippocampus is necessary for memory formation. Neurobiol Learn Mem 87: 303-307.

Blundell J, Kouser M, Powell CM. 2008. Systemic inhibition of mammalian target of rapamycin inhibits fear memory reconsolidation. Neurobiol Learn Mem 90: 28-35.

Cammalleri M, Lutjens R, Berton F, King AR, Simpson C, Francesconi W, Sanna PP. 2003. Time-restricted role for dendritic activation of the mTOR-p70S6K pathway in the induction of late-phase long-term potentiation in the CA1. Proc Natl Acad Sci 100: 14368-14373.

Casadio A, Martin KC, Giustetto M, Zhu H, Chen M, Bartsch D, Bailey CH, Kandel ER. 1999. A transient, neuron-wide form of CREB-mediated long-term facilitation can be stabilized at specific synapses by local protein synthesis. Cell 99: 221-237.

Cassella JV, Davis M. 1986. The design and calibration of a startle measurement system. Physiol Behav 36: 377-383.

Debiec J, Ledoux JE. 2004. Disruption of reconsolidation but not consolidation of auditory fear conditioning by noradrenergic blockade in the amygdala. Neuroscience 129: 267-272.

Grillon C, Cordova J, Morgan CA, Charney DS, Davis M. 2004. Effects of the beta-blocker propranolol on cued and contextual fear conditioning in humans. Psychopharmacology (Berl) 175: 342-352.

Lee HJ, Berger SY, Stiedl O, Spiess J, Kim JJ. 2001. Post-training injections of catecholaminergic drugs do not modulate fear conditioning in rats and mice. Neurosci Lett 303: 123-126.

McGaugh JL. 2000. Memory-a century of consolidation. Science 287: 248-251.

McNish KA, Gewirtz JC, Davis M. 1997. Evidence of contextual fear after lesions of the hippocampus: A disruption of freezing but not fear-potentiated startle. J Neurosci 17: 9353-9360.

McNish KA, Gewirtz JC, Davis M. 2000. Disruption of contextual freezing, but not contextual blocking of fear-potentiated startle, after lesions of the dorsal hippocampus. Behav Neurosci 114: 64-76.

Missanin JR, Miller RR, Lewis DJ. 1968. Retrograde amnesia produced by electroconvulsive shock after activation of a consolidated memory trace. Science 160: $554-555$.

Nader K, Schafe GE, LeDoux JE. 2000. Fear memories require protein synthesis in the amygdala for reconsolidation after retrieval. Nature 406: $722-726$.

Parsons RG, Gafford GM, Helmstetter FJ. 2006. Translational control via the mammalian target of rapamycin pathway is critical for the formation and stability of long-term fear memory in amygdala neurons. J Neurosci 26: 12977-12983.

Paschall GY, Davis M. 2002. Olfactory-mediated fear-potentiated startle. Behav Neurosci 116: 4-12.

Plas DR, Thomas G. 2009. Tubers and tumors: Rapamycin therapy for benign and malignant tumors. Curr Opin Cell Biol 21: 230-236.

Raught B, Gingras AC, Sonenberg N. 2001. The target of rapamycin (TOR) proteins. Proc Natl Acad Sci 98: 7037-7044.

Tang SJ, Reis G, Kang H, Gingras AC, Sonenberg N, Schuman EM. 2002. A rapamycin-sensitive signaling pathway contributes to long-term synaptic plasticity in the hippocampus. Proc Natl Acad Sci 99: 467-472.

Thomson LM, Sutherland RJ. 2005. Systemic administration of lipopolysaccharide and interleukin-1beta have different effects on memory consolidation. Brain Res Bull 67: 24-29.

Tischmeyer W, Schicknick H, Kraus M, Seidenbecher CI, Staak S, Scheich H, Gundelfinger ED. 2003. Rapamycin-sensitive signalling in long-term consolidation of auditory cortex-dependent memory. Eur J Neurosci 18: 942-950.

Received June 16, 2010; accepted in revised form August 24, 2010. 


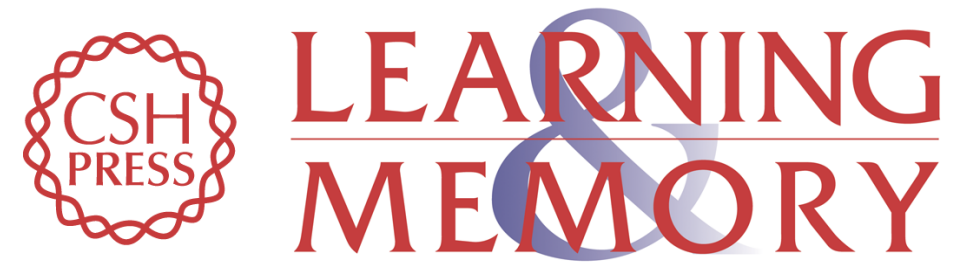

\section{Differing effects of systemically administered rapamycin on consolidation and reconsolidation of context vs. cued fear memories}

Ebony M. Glover, Kerry J. Ressler and Michael Davis

Learn. Mem. 2010, 17:

Access the most recent version at doi:10.1101/lm.1908310

References This article cites 20 articles, 7 of which can be accessed free at: http://learnmem.cshlp.org/content/17/11/577.full.html\#ref-list-1

License

Email Alerting

Receive free email alerts when new articles cite this article - sign up in the box at the Service top right corner of the article or click here. 